\title{
Analysis of a Model with Multiple Infectious Stages and Arbitrarily Distributed Stage Durations
}

\author{
Y. Yang ${ }^{a}$, D. $\mathrm{Xu}^{b}$ and Z. Feng ${ }^{a 1}$ \\ ${ }^{a}$ Department of Mathematics, Purdue University, West Lafayette, IN 47907, USA \\ ${ }^{b}$ Department of Mathematics, Southern Illinois University, Carbondale, IL 62901, USA
}

\begin{abstract}
Infectious diseases may have multiple infectious stages with very different epidemiological attributes, including infectivity and disease progression. These stages are often assumed to have exponentially distributed durations in epidemiological models. However, models that use the exponential distribution assumption (EDA) may generate biased and even misleading results in some cases. This discrepancy is particularly damaging if the models are employed to assist policy-makers in disease control and interventions. This paper studies a mathematical model that includes multiple infectious stages and general distributions for the stage durations (with the exponential distribution as a special case). Formulas for the control reproductive number, $\mathcal{R}_{c}$, and the basic reproductive number, $\mathcal{R}_{0}$, are derived, which can be conveniently applied to models in which specific stage distributions are assumed. It is also shown that the disease dynamics are determined by the reproductive numbers.
\end{abstract}

Key words: epidemiological model, disease stage distribution, disease control AMS subject classification: 37N25, 92D30

\section{Introduction}

One of the most common assumptions used in epidemiological models is that the disease stages are exponentially distributed. That is, the probability that an individual will remain in a disease stage $s$ time units after entering the stage is described by an exponential function of the form $e^{-\theta s}$, where $\theta>0$ is a constant with $1 / \theta$ representing the mean period of staying

\footnotetext{
${ }^{1}$ Corresponding author. Email: zfeng@math.purdue.edu
} 
in the stage (see [6] for a more detailed discussion on disease stage distributions). This amounts to assuming that an individual transfers from one stage to the next at a constant per capita rate and, consequently, the disease transmission process can be modeled using ordinary differential equations. Although the exponential distribution assumption (EDA) is very helpful to simplify the models and often provides a good approximation, it has been shown that in some cases, models that use this assumption may produce misleading results in assessing the effectiveness of various disease control strategies [1]. The influence of the EDA in epidemiological models and the need to use more realistic distributions have also been studied by other researchers (see, for example, $[3,4,5,7]$ ).

The model considered in this paper is an extension of a model studied in [1], which is an $S E I R$ model with quarantine, isolation, and arbitrary distributions for the disease stages. The standard SEIR model has the form:

$$
\begin{aligned}
& d S / d t=\mu N-\beta S \frac{I}{N}-\mu S, \\
& d E / d t=\beta S \frac{I}{N}-(\alpha+\mu) E, \\
& d I / d t=\alpha E-(\delta+\mu) I, \\
& d R / d t=\delta I-\mu R .
\end{aligned}
$$

The variables $S$ (susceptible), $E$ (exposed but not infectious), $I$ (infectious), and $R$ (recovered) represent the numbers of individuals in the respective classes, and $N=S+E+I+R$ is the total population size. The parameters $\alpha$ and $\delta$ are the rates at which an individual leaves the $E$ and $I$ classes, respectively. The per-capita birth and natural death rates are equal denoted by $\mu$, and $\beta$ is the disease transmission rate.

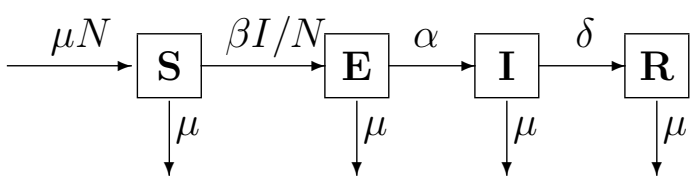

Fig.1 Disease transmission diagram for the SEIR model.

Several assumptions are made in Model (1.1). For the purpose of this paper, we will focus on two of them. First, there are two disease classes, $E$ and $I$. Only individuals in the $I$ class can transmit the disease, and all individuals in $I$ have the same infectivity, which is represented by the infection rate $\beta$. Second, the disease durations of the $E$ and $I$ stages are exponentially distributed with the mean latent period $1 / \alpha$ and the mean infectious period $1 / \delta$, respectively. Other assumptions include the following. (i) The population is homogeneous and the population size is large enough so that the force of infection takes the standard incidence from, $\beta S I / N$. (ii) The disease is not vital so that the disease-induced death rate is ignored (for example, childhood diseases such as chickenpox, scarlet fever, or mild strains of influenza). (iii) The population size remains constant for all time so that the per capita birth rate and per capita death rate are equal. (iv) The immunity obtained after recovering from the infection is permanent. 
In some diseases, an infectious individual may have very different infectivities at various disease stages due to the difference in viral load and/or activity level (e.g., HIV, TB, SARS), which may also lead to the variation in disease progression. This may have important implications for the questions to be considered using mathematical models, especially when the infectious period is long. For such diseases, the study of questions associated with disease intervention (e.g., treatment, isolation, etc.), it may be more appropriate to divide the single infectious stage $I$, as in Model (1.1), into multiple infectious stages so that different parameter values may be assigned to different stages. This is the main focus of this paper.

In this paper, we extend the model in [1] by including two infectious stages. Both quarantine and isolation are considered and a general distribution is assumed for each of the disease stages. Using the model, we derive an expression for the control reproductive number, $\mathcal{R}_{c}$, which reduces to the usual basic reproductive number, $\mathcal{R}_{0}$, when control measures are absent. These formulas can be quite useful as they are obtained for arbitrary distributions of the disease stages. When specific stage distributions are determined for a given disease, the formulas for $\mathcal{R}_{c}$ and $\mathcal{R}_{0}$ can be obtained by replacing the general distributions by specific ones. This is illustrated in an example in Section 3.2. We also show that $\mathcal{R}_{c}=1$ provides a threshold condition for determining whether or not the disease will die out.

\section{$2 \quad$ The model}

When quarantine and isolation are considered as measures for disease control, two additional classes, $Q$ (Quarantine) and $H$ (Hospitalization or isolation), can be introduced to the $S E I R$ model, as was done in [1]. It is assumed that an individual in $E$ can be quarantined and an individual in $I$ isolated according to certain distributions. The current model in this paper introduces two sub-classes, $I_{1}$ and $I_{2}$, of the infectious class $I$, and two corresponding sub-classes, $H_{1}$ and $H_{2}$, of the $H$ class. An example of such disease is influenza. The first infectious class, $I_{1}$, may represent the prodrome phase, while the second class, $I_{2}$, may represent the acute respiratory phase. These two stages have several important differences. For example, the average durations for these two stages are 4 and 8 days, respectively, and the infectivity at the respiratory phase is much higher than that in the prodrome phase. In addition, the probability of an individual in the $I_{2}$ stage seeking medical care is much higher that those in the $I_{1}$ stage. In our model, we assume that the stage durations for $I_{1}$ and $I_{2}$ can have different distributions, and the probabilities of being isolated from the two sub-stages can be different. The corresponding disease transmission diagram is shown in Fig. 2, in which the transition rates are described by general stage distribution functions whose definitions are given below. The birth and death rates are omitted in the diagram but are included in the model. As the focus of this model is on the role of arbitrarily distributed distributions of the disease stages, the per capita rates for birth and natural death are assumed to be constant, $\mu$ (i.e., the survival from natural death is given by an exponential function). 


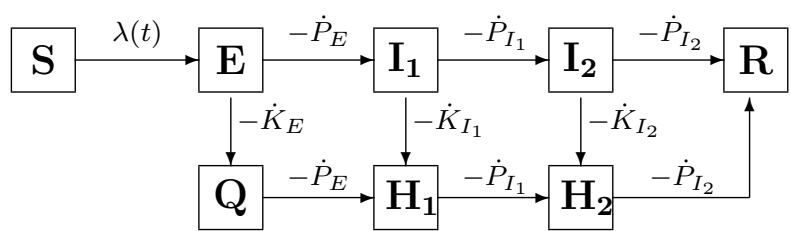

Fig. 2 Transmission diagram for two infectious stages and general stage distributions.

As in [1], let $P_{E}, P_{I_{1}}, P_{I_{2}}$ describe the durations of stages $E, I_{1}$ and $I_{2}$, respectively. That is, $P_{i}(s)\left(i=E, I_{1}, I_{2}\right)$ gives the probability of being still in the same stage at stage age $s$. These functions satisfy the properties

$$
P_{i}(0)=1, \quad \dot{P}_{i}(s) \leq 0, \quad \int_{0}^{\infty} P_{i}(s) d s<\infty, \quad i=E, I_{1}, I_{2} .
$$

A special case of (2.2) is when $P_{E}, P_{I_{1}}, P_{I_{2}}$ are all exponential distributions, for example,

$$
P_{E}(s)=e^{-\alpha s}, \quad P_{I_{1}}(s)=e^{-\delta_{1} s}, \quad P_{I_{2}}(s)=e^{-\delta_{2} s} .
$$

In this case, the constants $\alpha, \delta_{1}$ and $\delta_{2}$ represent the respective rates at which individuals move to the respective next stage $\left(E \rightarrow I_{1} \rightarrow I_{2} \rightarrow R\right)$, and the mean durations in these stages are $1 / \alpha, 1 / \delta_{1}$ and $1 / \delta_{2}$, respectively.

Let $K_{E}(s), K_{I_{1}}(s), K_{I_{2}}(s)$ denote the probabilities of not being quarantined or isolated at stage age $s$ from stages $E, I_{1}, I_{2}$, respectively. Then

$$
\bar{K}_{i}(s)=1-K_{i}(s), \quad i=E, I_{1}, I_{2}
$$

gives the probability of being quarantined or isolated from stage $i$.

Following the transition diagram in Fig. 2, the number of individuals who became exposed at some time $s \in(0, t)$ and are still alive and in the $E$ class at time $t$ is given by

$$
E(t)=\int_{0}^{t} \lambda(s) S(s) P_{E}(t-s) K_{E}(t-s) e^{-\mu(t-s)} d s+E_{0}(t)
$$

where $\lambda(s)$ is the force of infection (to be given), and $E_{0}(t)$ denotes individuals initially exposed who have moved into the $E$ class and are still alive at time $t$. Differentiating the above equation,

$$
\begin{aligned}
E^{\prime}(t)= & \int_{0}^{t} \lambda(s) S(s) \dot{P}_{E}(t-s) K_{E}(t-s) e^{-\mu(t-s)} d s \\
& +\int_{0}^{t} \lambda(s) S(s) P_{E}(t-s) \dot{K}_{E}(t-s) e^{-\mu(t-s)} d s+\lambda(t) S(t)-\mu E(t)+E_{0}^{\prime}(t)
\end{aligned}
$$

The first and the second terms provide inputs for the $I_{1}$ and the $Q$ equations, respectively. If an individual has entered the $I_{1}$ stage then the probability that the person is still in the $I_{1}$ 
stage $s$ time unite later is $P_{I_{1}}(s) K_{I_{1}}(s)$, i.e., neither of the following has happened at stage age $s: 1)$ has progressed to the next disease stage, in which case the person would enter the $I_{2}$ stage; and 2) has been isolated, in which case the person would enter the $H_{1}$ stage.

Following the above arguments and using the same approach as in [1], we can derive equations for other variables and obtain the following model

$$
\begin{aligned}
& S=\int_{0}^{t} \mu N e^{-\mu(t-s)} d s-\int_{0}^{t} \lambda(s) S(s) e^{-\mu(t-s)} d s+S_{0} e^{-\mu t}, \\
& E=\int_{0}^{t} \lambda(s) S(s) P_{E}(t-s) K_{E}(t-s) e^{-\mu(t-s)} d s+E_{0}(t), \\
& Q=\int_{0}^{t} \lambda(s) S(s) P_{E}(t-s) \bar{K}_{E}(t-s) e^{-\mu(t-s)} d s+Q_{0}(t) \\
& I_{1}=\int_{0}^{t} \int_{0}^{\tau} \lambda(s) S(s)\left[-\dot{P}_{E}(\tau-s) K_{E}(\tau-s)\right] P_{I_{1}}(t-\tau) K_{I_{1}}(t-\tau) e^{-\mu(t-s)} d s d \tau+I_{10}(t), \\
& H_{1}=\int_{0}^{t} \int_{0}^{\tau} \lambda(s) S(s)\left[-\dot{P}_{E}(\tau-s) K_{E}(\tau-s)\right] P_{I_{1}}(t-\tau) \bar{K}_{I_{1}}(t-\tau) e^{-\mu(t-s)} d s d \tau+ \\
& \int_{0}^{t} \int_{0}^{\tau} \lambda(s) S(s)\left[-\dot{P}_{E}(\tau-s) \bar{K}_{E}(\tau-s)\right] P_{I_{1}}(t-\tau) e^{-\mu(t-s)} d s d \tau+H_{10}(t), \\
& I_{2}=\int_{0}^{t} \int_{0}^{u} \int_{0}^{\tau} \lambda(s) S(s)\left[-\dot{P}_{E}(\tau-s) K_{E}(\tau-s)\right]\left[-\dot{P}_{I_{1}}(u-\tau) K_{I_{1}}(u-\tau)\right] \\
& \times P_{I_{2}}(t-u) K_{I_{2}}(t-u) e^{-\mu(t-s)} d s d \tau d u+I_{20}(t), \\
& H_{2}=\int_{0}^{t} \int_{0}^{u} \int_{0}^{\tau} \lambda(s) S(s)\left[-\dot{P}_{E}(\tau-s) K_{E}(\tau-s)\right]\left[-\dot{P}_{I_{1}}(u-\tau) K_{I_{1}}(u-\tau)\right] \\
& \times P_{I_{2}}(t-u) \bar{K}_{I_{2}}(t-u) e^{-\mu(t-s)} d s d \tau d u \\
& +\int_{0}^{t} \int_{0}^{u} \int_{0}^{\tau} \lambda(s) S(s)\left[-\dot{P}_{E}(\tau-s) \bar{K}_{E}(\tau-s)\right]\left[-\dot{P}_{I_{1}}(u-\tau)\right] \\
& \times P_{I_{2}}(t-u) e^{-\mu(t-s)} d s d \tau d u \\
& +\int_{0}^{t} \int_{0}^{u} \int_{0}^{\tau} \lambda(s) S(s)\left[-\dot{P}_{E}(\tau-s) K_{E}(\tau-s)\right]\left[-\dot{P}_{I_{1}}(u-\tau) \bar{K}_{I_{1}}(u-\tau)\right] \\
& \times P_{I_{2}}(t-u) e^{-\mu(t-s)} d s d \tau d u+H_{20}(t), \\
& R=\int_{0}^{t} \int_{0}^{u} \int_{0}^{\tau} \lambda(s) S(s)\left[-\dot{P}_{E}(\tau-s)\right]\left[-\dot{P}_{I_{1}}(u-\tau)\right]\left[1-P_{I_{2}}(t-u)\right] e^{-\mu(t-s)} d s d \tau d u,
\end{aligned}
$$

where $N=S+E+Q+\sum_{i=1}^{2}\left(I_{i}+H_{i}\right)+R$ and $\lambda(t)$ is the force of infection given by

$$
\lambda(t)=\frac{1}{N}\left[\beta_{E}\left(E+\left(1-\rho_{E} Q\right)\right)+\sum_{i=1}^{2} \beta_{i}\left(I_{i}+\left(1-\rho_{i}\right) H_{i}\right)\right] .
$$

Clearly, the contributions to $\lambda(t)$ may come from all disease related variables: $E, Q, I_{i}$ and $H_{i}(i=1,2)$, with possibly different transmission rates $\left(\beta_{E}\right.$ and $\beta_{i}$ for $\left.i=1,2\right)$ and different reduction in infectivity due to quarantine $\left(\rho_{E}\right)$ and isolation $\left(\rho_{i}, i=1,2\right)$. Here, we allow 
individuals in the $E$ class to also be able to transmit the disease, possibly at a reduced rate (i.e., $\beta_{E} \leq \beta_{i}, i=1,2$ ). Of course, such transmission will not occur when $\beta_{E}=0$. The constant $\rho_{E}\left(0 \leq \rho_{E} \leq 1\right)$ represents the reduction in transmission from quarantine individuals $(Q)$, in comparison with non-quarantined $(E)$. Similarly, $\rho_{i}\left(i=1,2,0 \leq \rho_{i} \leq 1\right)$ is a measure for the efficiency of isolation, which reflects the difference in the transmission rates between isolated individuals $\left(H_{i}\right)$ and non-isolated $\left(I_{i}\right) . S_{0}$ is a constant and the term $X_{0}(t)\left(X=E, Q, I_{1}\right.$, etc.) at the end of the equations account for individuals who were present initially (may be in a different class), and $X_{0}(t) \rightarrow 0$ as $t \rightarrow \infty$. All variables and parameters are listed in Table 1 . We remark that, as illustrated in [1], the above system (2.4) reduces to a system of ordinary differential equations when $P_{i}(s)\left(i=E, I_{1}, I_{2}\right)$ are either exponential or gamma distributions, with $K_{i}(s)\left(i=E, I_{1}, I_{2}\right)$ being exponential functions.

To show that the model (2.4) is well-posed, we only need to show that $S(t)$ and $\lambda(t)$ remain nonnegative for all $t>0$ for positive initial conditions (as the parameter functions satisfy $0 \leq P_{i} \leq 1,0 \leq K_{i} \leq 1$, and $\dot{P}_{i} \leq 0$ for $\left.i=E, I_{1}, I_{2}\right)$. Let $S(0)>0$ and $\lambda(0)>0$. Suppose that $\hat{t}$ is the time at which $S(\hat{t})=0$ with

$$
S(t)>0, \quad \lambda(t)>0 \quad \text { for all } 0<t<\hat{t} .
$$

Then, from the equations in (2.4) we know that $E(\hat{t})>0, Q(\hat{t})>0, I_{i}(\hat{t})>0, H_{i}(\hat{t})>0$ $\left(i=I_{1}, I_{2}\right)$. Hence, $N(\hat{t})>0$. Differentiating the $S$ equation in $(2.4)$ we get

$$
d S / d t=\mu N-\mu S-\lambda S,
$$

from which we know that $d S(\hat{t}) / d t=\mu N(\hat{t})>0$. It follows that $S(t)$ will remain nonnegative for all $t>0$. Now suppose that $\tilde{t}$ is the time at which $\lambda(\tilde{t})=0$ with

$$
S(t)>0, \quad \lambda(t)>0 \quad \text { for all } 0<t<\tilde{t} .
$$

This implies that $E(\tilde{t})>0, Q(\tilde{t})>0, I_{i}(\tilde{t})>0, H_{i}(\tilde{t})>0\left(i=I_{1}, I_{2}\right)$. Since at least one of transmission rates, $\beta_{E}$ and $\beta_{i}(i=1,2)$, is not zero, we know from $(2.5)$ that $\lambda(\tilde{t})>0$. This contradicts to $\lambda(\tilde{t})=0$. Thus, $\lambda(t)>0$ for all $t>0$. It follows that the model (2.4) is well-posed.

\section{Analysis of the model}

In this section, the equilibria of (2.4) and their stability are studied. The threshold condition for the stability of the disease-free equilibrium can be used to derive the control reproductive number $\mathcal{R}_{c}$ and the basic reproductive number $\mathcal{R}_{0}$.

\subsection{Equilibria and stability}

The system of integral equations (2.4) can have up to two equilibria. One is the disease-free equilibrium

$$
U=\left(S, E, Q, I_{1}, H_{1}, I_{2}, H_{2}\right)=(N, 0,0,0,0,0,0)
$$


Table 1: Definitions of frequently used symbols

\begin{tabular}{ll}
\hline Symbol & Definition $\left(i=E, I_{1}, I_{2}\right)$ \\
\hline$S(t)$ & Number of susceptible individuals at time $t$ \\
$E(t)$ & Number of exposed individuals at time $t$ \\
$Q(t)$ & Number of quarantined (exposed) individuals at time $t$ \\
$I_{1}(t), I_{2}(t)$ & Number of infectious individuals at time $t$ \\
$H_{1}(t), H_{2}(t)$ & Number of hospitalized (or isolated) infectious individuals at time $t$ \\
$R(t)$ & Number of recovered individuals at time $t$ \\
$N$ & Total population size (constant) \\
$\lambda(t)$ & Force of infection at time $t$ \\
$\beta, \beta_{i}$ & Transmission coefficient \\
$\alpha$ & Per capita rate at which latent individuals become infectious under the EDA \\
$\delta_{1}, \delta_{2}$ & Per capita rates of leaving the infectious stages 1 and 2 under the EDA \\
$\mu$ & Per capita natural death rate \\
$\rho_{i}$ & Efficiency of quarantine and isolation $\left(0 \leq \rho_{i} \leq 1\right)$ \\
$P_{i}(s)$ & Probability that disease stage $i$ lasts longer than $s$ time units \\
$K_{i}(s)$ & Probability of not being quarantined or isolated at stage age $s$ \\
$T_{i}$ & Probability of surviving stage $i$ and entering next stage: $\int_{0}^{\infty}\left[-\dot{P}_{i}(s)\right] e^{-\mu s} d t$ \\
$\mathcal{T}_{i}$ & Quarantine- or isolation-adjusted probability (similar to $\left.T_{i}\right):$ \\
& $\int_{0}^{\infty}\left[-\dot{P}_{i}(s) K_{i}(s)\right] e^{-\mu s} d t$ \\
$D_{i}$ & Mean duration of stage $i$ (adjusted by death): $\int_{0}^{\infty} P_{i}(s) e^{-\mu s} d t$ \\
$\mathcal{D}_{i}$ & Quarantine- or isolation-adjusted mean duration of stage $i:$ \\
& $\int_{0}^{\infty} P_{i}(s) K_{i}(s) e^{-\mu s} d t$ \\
& The basic reproductive number
\end{tabular}


which always exists. The other one is the endemic equilibrium which exists only under certain conditions as shown later in this section.

To study the stability of $U$, we introduce the following notation for ease of presentation: $a_{1}(s)=e^{-\mu s} P_{E}(s) K_{E}(s), a_{2}(s)=e^{-\mu s} P_{E}(s) \bar{K}_{E}(s), a_{3}(s)=e^{-\mu s} \int_{0}^{s}\left[-\dot{P}_{E}(s-\tau) K_{E}(s-\right.$ $\tau)] P_{I_{1}}(\tau) K_{I_{1}}(\tau) d \tau$

$$
\begin{aligned}
& a_{4}(s)=e^{-\mu s} \int_{0}^{s}\left[-\dot{P}_{E}(s-\tau) K_{E}(s-\tau)\right] P_{I_{1}}(\tau) \bar{K}_{I_{1}}(\tau) d \tau, \\
& a_{5}(s)=e^{-\mu s} \int_{0}^{s}\left[-\dot{P}_{E}(s-\tau) \bar{K}_{E}(s-\tau)\right] P_{I_{1}}(\tau) d \tau, \\
& a_{6}(s)=e^{-\mu s} \int_{0}^{s} \int_{0}^{s-u}\left[-\dot{P}_{E}(s-\tau-u) K_{E}(s-\tau-u)\right]\left[-\dot{P}_{I_{1}}(\tau) K_{I_{1}}(\tau)\right] P_{I_{2}}(u) K_{I_{2}}(u) d \tau d u, \\
& a_{7}(s)=e^{-\mu s} \int_{0}^{s} \int_{0}^{s-u}\left[-\dot{P}_{E}(s-\tau-u) K_{E}(s-\tau-u)\right]\left[-\dot{P}_{I_{1}}(\tau) K_{I_{1}}(\tau)\right] P_{I_{2}}(u) \bar{K}_{I_{2}}(u) d \tau d u, \\
& a_{8}(s)=e^{-\mu s} \int_{0}^{s} \int_{0}^{s-u}\left[-\dot{P}_{E}(s-\tau-u) K_{E}(s-\tau-u)\right]\left[-\dot{P}_{I_{1}}(\tau) \bar{K}_{I_{1}}(\tau)\right] P_{I_{2}}(u) d \tau d u, \\
& a_{9}(s)=e^{-\mu s} \int_{0}^{s} \int_{0}^{s-u}\left[-\dot{P}_{E}(s-\tau-u) \bar{K}_{E}(s-\tau-u)\right]\left[-\dot{P}_{I_{1}}(\tau)\right] P_{I_{2}}(u) d \tau d u .
\end{aligned}
$$

Recall that the $X_{0}(t)\left(X=S, E, Q, I_{1}\right.$, etc.) terms go to zero as time increases, and hence, can be ignored for the stability analysis. By interchanging the orders of integrations we can rewrite the equations for $E, Q, I_{1}, H_{1}, I_{2}, H_{2}$ in (2.4) as

$$
\begin{aligned}
& E=\int_{0}^{t} \lambda(s) S(s) a_{1}(t-s) d s, \quad Q=\int_{0}^{t} \lambda(s) S(s) a_{2}(t-s) d s, \\
& I_{1}=\int_{0}^{t} \lambda(s) S(s) a_{3}(t-s) d s, \\
& H_{1}=\int_{0}^{t} \lambda(s) S(s)\left(a_{4}(t-s)+a_{5}(t-s)\right) d s, \\
& I_{2}=\int_{0}^{t} \lambda(s) S(s) a_{6}(t-s) d s, \\
& H_{2}=\int_{0}^{t} \lambda(s) S(s)\left(a_{7}(t-s)+a_{8}(t-s)+a_{9}(t-s)\right) d s .
\end{aligned}
$$

Let

$$
\mathcal{R}_{c}=\int_{0}^{\infty} A(s) d s
$$

where

$$
\begin{aligned}
A(s)= & \beta_{E}\left(a_{1}(s)+\left(1-\rho_{E}\right) a_{2}(s)\right)+\beta_{1}\left(a_{3}(s)+\left(1-\rho_{1}\right)\left[a_{4}(s)+a_{5}(s)\right]\right) \\
& +\beta_{2}\left(a_{6}(s)+\left(1-\rho_{2}\right)\left[a_{7}(s)+a_{8}(s)+a_{9}(s)\right]\right) .
\end{aligned}
$$


It turns out that this quantity, $\mathcal{R}_{c}$, derived from mathematical analysis is actually the usual reproductive number for the disease in the presence of control. In the current notation as given by (3.7) and (3.8), the formula for $\mathcal{R}_{c}$ seems very complicated especially with the long expressions of $a_{i}(s)(i=1, \cdots, 9)$. In the following section, we will provide another formulation of $\mathcal{R}_{c}$ using biologically meaningful notations, which will make it more useful in the discussion of disease control strategies.

As in most epidemiological models, the reproductive number $\mathcal{R}_{c}$ provides a threshold quantity which determines the disease dynamics, as shown in the following result for the global stability of the equilibrium $U$.

Theorem 1. The disease-free equilibrium $U$ is a global attractor if $\mathcal{R}_{c}<1$.

A proof of this result can be found in the appendix. Result 1 implies that the disease will die out under a control program for which $\mathcal{R}_{c}<1$. Another representation of $\mathcal{R}_{c}$ with a more clear biological interpretation is given in Section 3.2.

To find an endemic equilibrium, we first use the $a_{i}(s)(i=1,2, \cdots, 9)$ expressions to rewrite $\lambda(t)($ see $(2.5))$ as

$$
\lambda(t)=\frac{1}{N} \int_{0}^{t} \lambda(s) S(s) A(t-s) d s,
$$

where the function $A(s)$ is given in (3.8). Integrating the $S$ equation in (2.4), we have

$$
S(t)=N-\int_{0}^{t} \lambda(s) S(s) e^{-\mu(t-s)} d s .
$$

Observe that the two equations, (3.9) and (3.10), are independent of variables other than $\lambda$ and $S$ ( $N$ is consistent). From the equations in (2.4) we see that all disease variables will go to zero if $\lambda(t) \rightarrow 0$ as $t \rightarrow \infty$. Thus, the variable $\lambda$ can be used to determine whether the disease will die out or becomes endemic. This allows us to reduce the problem to the study of the system of two equations involving only $\lambda$ and $S$ given in (3.9) and (3.10), respectively.

Let $\left(S^{*}, \lambda^{*}\right)$ denote an endemic equilibrium $\left(\lambda^{*}>0\right)$ of the $(S, \lambda)$ system. Then $S^{*}$ and $\lambda^{*}$ satisfy the equations

$$
\begin{aligned}
& S^{*}=N-\int_{0}^{\infty} \lambda^{*} S^{*} e^{-\mu s} d s, \\
& \lambda^{*}=\frac{1}{N} \int_{0}^{\infty} \lambda^{*} S^{*} A(s) d s,
\end{aligned}
$$

from which we can obtain the unique endemic equilibrium:

$$
\left(S^{*}, \lambda^{*}\right)=\left(\frac{N}{\mathcal{R}_{c}}, \mu\left(\mathcal{R}_{c}-1\right)\right) .
$$

Following the same arguments as in [1], we can prove the following result.

Theorem 2. If $\mathcal{R}_{c}>1$, then the unique endemic equilibrium $\left(S^{*}, \lambda^{*}\right)$ exists and is locally asymptotically stable. In this case, the disease-free equilibrium is unstable. 
We omit the proof of Result 2 as it is fairly similar to the proof for the same result in [1]. Results 1 and 2 suggest that the quantity $\mathcal{R}_{c}$ can be very helpful for evaluating disease control strategies.

\subsection{The reproductive numbers $\mathcal{R}_{c}$ and $\mathcal{R}_{0}$}

As shown in Section 3.1, the quantity $\mathcal{R}_{c}$ provides a threshold condition for disease control. We now provide an interpretation of the quantity to show that $\mathcal{R}_{c}$ is indeed the reproductive number for the disease in the presence of disease control.

Note that the integrals

$$
T_{E}=\int_{0}^{\infty}\left[-\dot{P}_{E}(s)\right] e^{-\mu s} d s, \quad T_{I_{1}}=\int_{0}^{\infty}\left[-\dot{P}_{I_{1}}(s)\right] e^{-\mu s} d s
$$

represent the probabilities that an individual survives natural death in the stages $E$ and $I_{1}$, respectively, and enters the next stage (i.e., $E \rightarrow I_{1} \rightarrow I_{2}$ ); whereas the integrals

$$
\mathcal{T}_{E}=\int_{0}^{\infty}\left[-\dot{P}_{E}(s) K_{E}(s)\right] e^{-\mu s} d s, \quad \mathcal{T}_{I_{1}}=\int_{0}^{\infty}\left[-\dot{P}_{I_{1}}(s) K_{I_{1}}(s)\right] e^{-\mu s} d s
$$

represent the probabilities that an individual survives natural death and is not quarantined or isolated in the stages $E$ and $I_{1}$, respectively, and enters the next stage $\left(E \rightarrow I_{1} \rightarrow I_{2}\right)$. Let $D_{i}$ denote the mean duration that an individual stays in the stage $i$, and let $\mathcal{D}_{i}$ denote the control-adjusted (i.e., quarantine or isolation) mean duration that an individual stays in the stage $i\left(i=E, I_{1}, I_{2}\right)$. Then

$$
\begin{array}{ll}
D_{E}=\int_{0}^{\infty} P_{E}(s) e^{-\mu s} d s, & \mathcal{D}_{E}=\int_{0}^{\infty} P_{E}(s) K_{E}(s) e^{-\mu s} d s \\
D_{I_{1}}=\int_{0}^{\infty} P_{I_{1}}(s) e^{-\mu s} d s, & \mathcal{D}_{I_{1}}=\int_{0}^{\infty} P_{I_{1}}(s) K_{I_{1}}(s) e^{-\mu s} d s \\
D_{I_{2}}=\int_{0}^{\infty} P_{I_{2}}(s) e^{-\mu s} d s, & \mathcal{D}_{I_{2}}=\int_{0}^{\infty} P_{I_{2}}(s) K_{I_{2}}(s) e^{-\mu s} d s .
\end{array}
$$

Using the integrals in (3.11)-(3.13) and their biological meanings, we know that the quantities

$$
\begin{aligned}
& \mathcal{R}_{E}=\beta_{E} \int_{0}^{\infty} a_{1}(s) d s=\beta_{E} \mathcal{D}_{E}, \\
& \mathcal{R}_{I_{1}}=\beta_{1} \int_{0}^{\infty} a_{3}(s) d s=\beta_{1} \mathcal{T}_{E} \mathcal{D}_{I_{1}}, \\
& \mathcal{R}_{I_{2}}=\beta_{2} \int_{0}^{\infty} a_{6}(s) d s=\beta_{2} \mathcal{T}_{E} \mathcal{T}_{I_{1}} \mathcal{D}_{I_{2}}
\end{aligned}
$$

give the numbers of secondary infections by an individual in the $E, I_{1}$ and $I_{2}$ stages, respectively, who did not become quarantined or isolated. 
Similarly, for an individual who is in $H_{1}$ by isolation, and an individual who is in $H_{2}$ by isolation, and an individual who entered $H_{2}$ from $H_{1}$, the numbers of secondary infections are given respectively by

$$
\begin{aligned}
& \mathcal{R}_{I_{1} H_{1}}=\beta_{1}\left(1-\rho_{1}\right) \int_{0}^{\infty} a_{4}(s) d s=\beta_{1}\left(1-\rho_{1}\right) \mathcal{T}_{E}\left(D_{I_{1}}-\mathcal{D}_{I_{1}}\right) \\
& \mathcal{R}_{I_{2} H_{2}}=\beta_{2}\left(1-\rho_{2}\right) \int_{0}^{\infty} a_{7}(s) d s=\beta_{2}\left(1-\rho_{2}\right) \mathcal{T}_{E} \mathcal{T}_{I_{1}}\left(D_{I_{2}}-\mathcal{D}_{I_{2}}\right) \\
& \mathcal{R}_{H_{1} H_{2}}=\beta_{2}\left(1-\rho_{2}\right) \int_{0}^{\infty}\left(a_{8}(s)+a_{9}(s)\right) d s=\beta_{2}\left(1-\rho_{2}\right)\left(T_{E} T_{I_{1}}-\mathcal{T}_{E} \mathcal{T}_{I_{1}}\right) D_{I_{2}} .
\end{aligned}
$$

Finally, for an individual in the $Q$ class and an individual who entered $H_{1}$ from $Q$, the numbers of secondary infections are given respectively by

$$
\begin{aligned}
& \mathcal{R}_{Q}=\beta_{E}\left(1-\rho_{E}\right) \int_{0}^{\infty} a_{2}(s) d s=\beta_{E}\left(1-\rho_{E}\right)\left(D_{E}-\mathcal{D}_{E}\right), \\
& \mathcal{R}_{Q H_{1}}=\beta_{1}\left(1-\rho_{1}\right) \int_{0}^{\infty} a_{5}(s) d s=\beta_{1}\left(1-\rho_{1}\right)\left(T_{E}-\mathcal{T}_{E}\right) D_{I_{1}} .
\end{aligned}
$$

Since $D_{i} \geq \mathcal{D}_{i}$ and $T_{i} \geq \mathcal{T}_{i}$ for $i=E, I_{1}, I_{2}$, we have $\mathcal{R}_{j} \geq 0$ for all $j=E, Q, I_{1}, I_{2}, I_{1} H_{1}$, $I_{2} H_{2}, H_{1} H_{2}$. Then, the sum of these quantities gives the overall reproductive number, which is exactly the quantity $\mathcal{R}_{c}$ given in (3.7). Thus, we can rewrite $\mathcal{R}_{c}$ as

$$
\mathcal{R}_{c}=\mathcal{R}_{E}+\mathcal{R}_{Q}+\mathcal{R}_{I_{1}}+\mathcal{R}_{I_{2}}+\mathcal{R}_{Q H_{1}}+\mathcal{R}_{I_{1} H_{1}}+\mathcal{R}_{I_{2} H_{2}}+\mathcal{R}_{H_{1} H_{2}}
$$

It is clear that $\mathcal{R}_{c}$ is indeed the control reproductive number.

If control measures are absent, then $K_{i}(s)=1$ for all $s$ and $i=E, I_{1}, I_{2}$. In this case, $D_{i}=\mathcal{D}_{i}$ and $T_{i}=\mathcal{T}_{i}$ for $i=E, I_{1}, I_{2}$. Hence, $\mathcal{R}_{c}$ reduces to the usual basic reproductive number

$$
\mathcal{R}_{0}=\beta_{E} D_{E}+\beta_{1} T_{E} D_{I_{1}}+\beta_{2} T_{E} T_{I_{1}} D_{I_{2}} .
$$

Next, we use an example to illustrate the use of the formulas for $\mathcal{R}_{c}$ and $\mathcal{R}_{0}$. The example is for the case when the distributions $P_{i}\left(i=E, I_{1}, I_{2}\right)$ are exponential.

Example. Let $P_{i}$ be exponential distributions as given in (2.3) for $i=E, I_{1}$, and $I_{2}$. In this case, the positive constants, $\alpha, \delta_{1}$ and $\delta_{2}$, represent respectively the rates at which individuals leave the $E, I_{1}$ and $I_{2}$ classes. Let the functions $K_{i}\left(i=E, I_{1}, I_{2}\right)$ be given in the forms: $K_{E}(s)=e^{-q s}, K_{I_{1}}(s)=e^{-h_{1} s}, K_{I_{2}}(s)=e^{-h_{2} s}$, where $q, h_{1}, h_{2}$ are positive constants representing the rates of quarantine, isolation from $I_{1}$, and isolation from $I_{2}$, respectively. In this case, the eight components of $\mathcal{R}_{c}$, given in (3.14)-(3.16), can be written as the following:

$$
\begin{aligned}
& \mathcal{R}_{E}=\frac{\beta_{E}}{q+\alpha+\mu}, \quad \mathcal{R}_{Q}=\frac{\beta_{E}\left(1-\rho_{E}\right) q}{(\alpha+\mu)(q+\alpha+\mu)}, \quad R_{Q H_{1}}=\frac{\beta_{1}\left(1-\rho_{1}\right) \alpha q}{(\alpha+\mu)(q+\alpha+\mu)\left(\delta_{1}+\mu\right)}, \\
& \mathcal{R}_{I_{1}}=\frac{\beta_{1} \alpha}{(q+\alpha+\mu)\left(h_{1}+\delta_{1}+\mu\right)}, \quad \mathcal{R}_{I_{2}}=\frac{\beta_{2} \alpha \delta_{1}}{(q+\alpha+\mu)\left(h_{1}+\delta_{1}+\mu\right)\left(h_{2}+\delta_{2}+\mu\right)},
\end{aligned}
$$




$$
\begin{aligned}
\mathcal{R}_{I_{1} H_{1}} & =\frac{\beta_{1}\left(1-\rho_{1}\right) \alpha h_{1}}{(q+\alpha+\mu)\left(h_{1}+\delta_{1}+\mu\right)\left(\delta_{1}+\mu\right)}, \\
\mathcal{R}_{I_{2} H_{2}} & =\frac{\beta_{2}\left(1-\rho_{2}\right) \alpha \delta_{1} h_{2}}{(q+\alpha+\mu)\left(h_{1}+\delta_{1}+\mu\right)\left(h_{2}+\delta_{2}+\mu\right)\left(\delta_{2}+\mu\right)}, \\
\mathcal{R}_{H_{1} H_{2}} & =\frac{\beta_{2}\left(1-\rho_{2}\right) \alpha \delta_{1}\left(q h_{1}+q\left(\delta_{1}+\mu\right)+h_{1}(\alpha+\mu)\right)}{(q+\alpha+\mu)\left(h_{1}+\delta_{1}+\mu\right)(\alpha+\mu)\left(\delta_{1}+\mu\right)\left(\delta_{2}+\mu\right)} .
\end{aligned}
$$

Substitution of the above expressions for $\mathcal{R}_{j}$ in (3.17) yields the expression of $\mathcal{R}_{c}$. In the absent of control, $q=h_{1}=h_{2}=0$, and the basic reproductive number is

$$
\mathcal{R}_{0}=\frac{\beta_{E}}{\alpha+\mu}+\frac{\beta_{1} \alpha}{(\alpha+\mu)\left(\delta_{1}+\mu\right)}+\frac{\beta_{2} \alpha \delta_{1}}{(\alpha+\mu)\left(\delta_{1}+\mu\right)\left(\delta_{2}+\mu\right)} .
$$

Since the formula for $\mathcal{R}_{c}$ contains all the parameters representing disease control $\left(\chi, \phi_{i}\right.$, $\left.\rho_{i}, i=1,2\right)$, it can be used to explore the effect of the corresponding control measures in the reduction of the reproductive number from $\mathcal{R}_{0}$. It is shown in [1] that models with exponentially distributed disease durations may generate misleading results when used to compare the effectiveness of control strategies. One way to improve the models is to replace the exponential distribution by more realistic distributions, one of which is the gamma distribution. We have considered in [2] the case when $P_{i}\left(i=E, I_{1}, I_{2}\right)$ are gamma distributions.

\section{Discussion}

In this paper, we extended the model in [1] by including multiple infectious stages. The current model allows the durations of disease stages to be arbitrarily distributed, which includes the exponential distribution as a special case. The model also includes quarantine and isolation for infected individuals so that it can be used for the study of disease control strategies. The derivation of the reproductive number in the presence of disease control $\left(\mathcal{R}_{c}\right)$ and in the absence of control $\left(\mathcal{R}_{0}\right)$ provides formulas for general stage distributions. We have also established that the disease will die out if and only if $\mathcal{R}_{c}<1$.

As the formulas for $\mathcal{R}_{c}$ and $\mathcal{R}_{0}$ in (3.17) and (3.18) are derived for arbitrarily distributed stage durations, they provide immediate expressions of the reproductive numbers for models in which specific stage distributions are known (exponential, gamma, etc.), as illustrated in the example in Section 3.2.

\section{Appendix}

In this Appendix, we provide a proof of Result 1. First we notice that the $X_{0}\left(X=S, E, Q, I_{1}\right.$ etc.) terms in the equations in (2.4) can be ignored. Note also that the $R$ equation in the 
system (2.4) can be rewritten as

$$
R=\int_{0}^{t} \int_{0}^{t-s} \int_{0}^{t-s-u} \lambda(s) S(s)\left[-\dot{P}_{E}(t-s-u-\tau)\right]\left[-\dot{P}_{I_{1}}(\tau)\right]\left[1-P_{I_{2}}(u)\right] d \tau d u d s .
$$

Then using the above equation, together with the $S$ equation in (2.4) and the equations in (3.6) we can show that the total population $N$ satisfies the equation

$$
N(t)=\int_{0}^{t} \mu N(s) e^{-\mu(t-s)} d s
$$

from which we get

$$
d N / d t=-\mu N(t)+\mu N(t)=0 .
$$

Thus, $N$ remains constant for all $t>0$. Then, using (2.5), (3.6), and $S(t) \leq N(t)$, we can rewrite $\lambda(t)$ as follows

$$
\lambda(t)=\frac{1}{N} \int_{0}^{t} \lambda(s) S(s) A(t-s) d s \leq \int_{0}^{t} \lambda(s) A(t-s) d s,
$$

where $A(s)$ is given in (3.8). Notice that $\lambda(t)$ is bounded by $\beta=\max \left\{\beta_{E}, \beta_{1}, \beta_{2}\right\}$ on $[0, \infty)$. Let $\lambda^{\infty}=\lim _{t \rightarrow \infty} \Lambda(t)$ with $\Lambda(t)=\sup _{s \geq t} \lambda(s)$. Then, there exists a sequence $t_{n} \rightarrow \infty$ as $n \rightarrow \infty$, such that $\lim _{n \rightarrow \infty} \lambda\left(t_{n}\right)=\lambda^{\infty}$. Assume that $t_{n+1}-t_{n} \rightarrow \infty$ as $n \rightarrow \infty$ (otherwise we can choose a subsequence). Then from (5.20) we have

$$
\begin{aligned}
\lambda\left(t_{n+1}\right) & \leq \int_{0}^{t_{n}} \lambda(s) A\left(t_{n+1}-s\right) d s+\int_{t_{n}}^{t_{n+1}} \lambda(s) A\left(t_{n+1}-s\right) d s \\
& \leq \beta \int_{t_{n+1}-t_{n}}^{t_{n+1}} A(s) d s+\Lambda\left(t_{n}\right) \int_{t_{n}}^{t_{n+1}} A\left(t_{n+1}-s\right) d s \\
& \leq \beta \int_{t_{n+1}-t_{n}}^{t_{n+1}} A(s) d s+\Lambda\left(t_{n}\right) \int_{0}^{t_{n+1}-t_{n}} A(s) d s .
\end{aligned}
$$

From the convergence of $\int_{0}^{\infty} A(s) d s$, we know that the first integral in (5.21) goes to zero as $n \rightarrow \infty$, and hence the inequality (5.21) reduces to $\lambda^{\infty} \leq \lambda^{\infty} \mathcal{R}_{c}$ as $n \rightarrow \infty$. Therefore, if $\mathcal{R}_{c}<1$ then $\lambda^{\infty}=0$. That is, $\lim _{t \rightarrow \infty} \lambda(t)=0$. Hence $E(t), Q(t), I_{1}(t), H_{1}(t), I_{2}(t)$ and $H_{2}(t)$ converge to zero while $S(t)$ goes to $N$ as $t$ goes to infinity. The global attractivity of $U$ is obtained.

\section{Acknowledgements}

This research is supported in part by the NSF grants DMS-0719783 to DX and DMS-0719697 to ZF. The authors are grateful to the reviewer for very helpful suggestions that greatly improved the paper. 


\section{References}

[1] Z. Feng, D. Xu, H. Zhao. Epidemiological models with non-exponentially distributed disease stages and applications to disease control. Bull. Math. Biol., 69 (2007), No. 5, 1511-1536.

[2] Z. Feng, Y. Yang, D. Xu, P. Zhang, M McCauley, J. Glasser. Timely identification of control strategies for emerging infectious diseases: Severe acute respiratory syndrome in Singapore. Submitted.

[3] H. Hethcote, D. Tudor. Integral equation models for endemic infectious diseases. J. Math. Biol., 9 (1980), 37-47.

[4] M.J. Keeling, P. Rohani. Modeling infectious diseases in humans and animals. Princeton University Press. Princeton, 2007.

[5] A. Lloyd. Realistic distributions of infectious periods in epidemic models. Theor. Pop. Biol., 60 (2001), 59-71.

[6] H. Thieme. Mathematics in population biology. Princeton University Press, Princeton, 2003.

[7] H.J. Wearing, P. Rohani, M.J. Keeling. Appropriate models for the management of infectious diseases. PLoS Medicine, 2 (2005), No. 7, 621-627. 